\section{COVID-19 y comportamiento psicológico: revisión sistemática de los efectos psicológicos de las pandemias del siglo XXI}

\author{
DAVID CUADRA-MARTÍNEZ ${ }^{1, \mathrm{a}, \mathrm{b}}$, PABLO J. CASTRO-CARRASCO ${ }^{2,5,6, a, \mathrm{c}}$, \\ JOSÉ SANDOVAL-DÍAZ ${ }^{3, \mathrm{a}, \mathrm{c}}$, DANIEL PÉREZ-ZAPATA ${ }^{4, \mathrm{a}, \mathrm{b}}$, \\ DIEGO MORA DABANCENS ${ }^{1, \mathrm{a}, \mathrm{b}}$
}

\section{COVID-19 and psychological behavior: a systematic review of the psychological effects of 21 st century pandemics}

The aim of this work was to conduct a systematic review on psychological behavior in the context of pandemic scenarios during the twenty-first century. We focused on empirical works and brief case reports of H1N1 flu and COVID-19. Our review included 32 papers published both in English or Spanish. We built a set of tables that allowed us to classify the information in four main categories, namely the psychological impact of the pandemic, whether people follow or not official measures to protect themselves against the pandemic, psychological adherence considerations implied as mediators to respect official strategies, and relevant methodological characteristics of the pandemic research. Results show that there are significant impacts on the psychological behavior of people, social groups and organizations in several dimensions, namely emotion, cognition, behavior, mental health, organization and psychosocial factors. In addition, we found that certain social groups experienced a critical psychosocial impact likely due to the pandemic. Psychosocial factors affecting adherence were also identified, which allow us to better understand how health strategies are followed by the population. Different psychosocial suggestions, which emerged from the papers reviewed, were systematized and should be considered as possible pandemic strategies to be implemented.

(Rev Med Chile 2020; 148: 1139-1154)

Key words: Behavior; Pandemics; Psychology.
'Departamento de Psicología,

Facultad de Humanidades y

Educación, Universidad de

Atacama. Copiapó, Chile.

${ }^{2}$ Departamento de Psicología,

Facultad de Humanidades,

Universidad de La Serena. La

Serena, Chile.

${ }^{3}$ Departamento de Ciencias

Sociales, Universidad del Bío-Bío.

Chillán, Chile.

${ }^{4}$ Escuela de Psicología y Filosofía, Universidad de Tarapacá. Arica,

Chile.

5Universidad Católica del Maule.

Talca, Chile.

${ }^{6}$ Instituto Milenio para la

Investigación en Depresión

y Personalidad (MIDAP).

Investigador colaborador.

assicólogo.

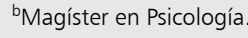

'Doctor en Psicología.

Trabajo no recibió financiamiento. Los autores declaran no tener conflictos de interés.

Recibido el 1 de marzo de 2020, aceptado el 10 de septiembre de 2020.

Correspondencia a: David Cuadra Martínez Copayapu 485. Copiapó, Chile. david.cuadra@uda.cl
E 111 de marzo del año 2020, la Organización Mundial de la Salud ${ }^{1}$ declaró estado de pandemia por COVID-19. El rápido avance de esta enfermedad altamente contagiosa ha sido calificado por los gobernantes y la prensa internacional como la crisis sanitaria más grave del último siglo. Esta crisis sanitaria global ha llevado a los gobiernos a establecer medidas urgentes con el fin de proteger a la población y frenar el avance de la enfermedad. Sin embargo, la afectación de la salud poblacional, el deterioro social debido a las medidas de aislamiento, el impacto económico y el estancamiento de los distintos servicios sociales (como la educación) ha sido evidente y de proporciones abismantes a nivel planetario.

El objetivo de la presente revisión es sistematizar el conocimiento científico que se tiene a la fecha sobre el comportamiento psicológico en 
situación de pandemia, a partir de los estudios empíricos y de reportes breves de caso, en el contexto de las pandemias declaradas durante el siglo XXI: la influenza H1N1 y el COVID-19. En este trabajo definimos comportamiento psicológico pandémico como todas las reacciones cognitivas, emocionales y conductuales de las personas, grupos sociales y organizaciones, observables y no observables, frente a la situación de amenaza o manifestación de una pandemia, incluyendo la respuesta psicológica para hacer frente a una enfermedad.

\section{$\underline{\text { Psicología de la pandemia }}$}

La historia muestra que, durante las pandemias ocurridas, los políticos, científicos, académicos y profesionales no solo han discutido y propuesto medidas para el cuidado de la salud física, sino que también sobre nuevas formas psicosociales de convivir bajo el contexto de emergencia ${ }^{2}$, lo que ha impactado decididamente en el estado psicológico de las personas, grupos y organizaciones.

De hecho, una de las consecuencias más importantes de una pandemia es el surgimiento o aumento de trastornos, de emociones negativas tales como ansiedad, miedo, inseguridad, incertidumbre ${ }^{3} \mathrm{y}$, en general, de preocupación en los ciudadanos en todo el mundo ${ }^{4}$. La teoría del sistema inmune conductual (i.e., Behavioral Immune System, BIS por sus siglas en inglés) señala que las personas despliegan una serie de mecanismos cognitivos, emocionales y conductuales para enfrentar enfermedades contagiosas 5 . Junto a la teoría del estrés y la de evaluación del riesgo, estos modelos explican que en el contexto de una emergencia sanitaria las personas identifican claves con el fin de evitar exponerse al contagio (por ejemplo, la tos en un contexto de pandemia por influenza), desarrollando emociones negativas y creencias que pueden magnificar o minimizar el riesgo. También despliegan comportamientos de protección ante el riesgo de contagio, que incluso pueden transformarse en conductas discriminatorias. Dichos comportamientos influyen en la adherencia de las medidas de prevención, repercuten en un deterioro importante de la salud psicológica ${ }^{6} \mathrm{o}$ afectan la convivencia social.

El impacto psicológico de la población por una pandemia ha recibido la atención de investi- gadores y a la fecha hay reportados una cantidad importante de estudios que dan cuenta de un efecto considerable en la población que requiere ser atendido ${ }^{7}$. Sin embargo, hasta hoy no se cuenta con una revisión sistemática de estos aportes.

Dado que durante el siglo XXI el mundo se ha visto golpeado por dos pandemias en un corto período de tiempo, es necesario aprender de estas experiencias, por lo que contar con un cuerpo de evidencia científica que permita clarificar la afectación psicológica de la población durante una pandemia y cómo abordarla se torna imperativo. Es por esto indispensable que las decisiones políticas y profesionales frente a una pandemia planifiquen las respuestas psicosociales en base al acervo científico existente ${ }^{8}$.

\section{Método}

Se llevó a cabo una revisión sistemática de estudios que reportaron dimensiones psicológicas del comportamiento de las personas en situación de pandemias del siglo XXI. Para esto, nos basamos en la definición de revisión sistemática y estructura propuesta por el método de Cochrane ${ }^{9}$.

\section{Proceso de búsqueda de la literatura}

La búsqueda de los estudios fue realizada en las siguientes bases de datos electrónicas: Web of Science, SciELO y Scopus (Elsevier). Se consideró artículos correspondientes a investigaciones empíricas y reportes breves de caso, escritos en inglés o español. Los criterios de inclusión fueron: a) que la temática de estudio o parte de ella sea sobre el comportamiento psicológico en situación de pandemia, incluyendo trabajos sobre el COVID-19, H1N1 o pandemia en general; b) que el estudio fuera en formato de artículo de investigación científica; c) publicado desde el año 2009 a abril de 2020. Se excluyeron: a) investigaciones empíricas y reportes breves de caso publicados en otros medios, como libros, manuales, boletines o congresos; b) estudios psicológicos o que abordaran aspectos psicológicos de pandemias que se declararon antes del siglo XXI, como el VIH; c) artículos en donde no se identificó claramente la referencia a una pandemia o a su metodología y d) literatura gris, por los problemas de acceso a esta información. Se utilizó el operador boléano AND con el símbolo +. Los términos libres clave 
para realizar la búsqueda de trabajos en las bases de datos fueron: "psicología + pandemia" y en inglés "psychology + pandemic" (o nombre de pandemia: COVID-19, gripe/influenza H1N1; gripe porcina/Swine flu); "psicología + pandemia + investigación (cualitativa, cuantitativa, mixta)" y en inglés "psychology + pandemic + research (qualitative, quantitative, mixed)"; "apoyo psicológico + pandemia" y en inglés "psychological support + pandemic". En las bases de datos se aplicaron los límites de búsqueda "full text" y "abstract" (93,5\% de los artículos fue encontrado a texto completo).
La Figura 1 muestra el proceso de búsqueda y selección de los artículos revisados.

La Tabla 1 muestra los trabajos analizados. Se encontraron 32 artículos que cumplieron los criterios de inclusión y su análisis de datos se llevó a cabo en base a una matriz que permitió analizar los artículos considerando las siguientes categorías: el impacto psicológico de la pandemia, la adherencia a las medidas para prevenir o tratar la pandemia, las consideraciones psicológicas para el abordaje de la pandemia y la metodología utilizada por los estudios revisados.

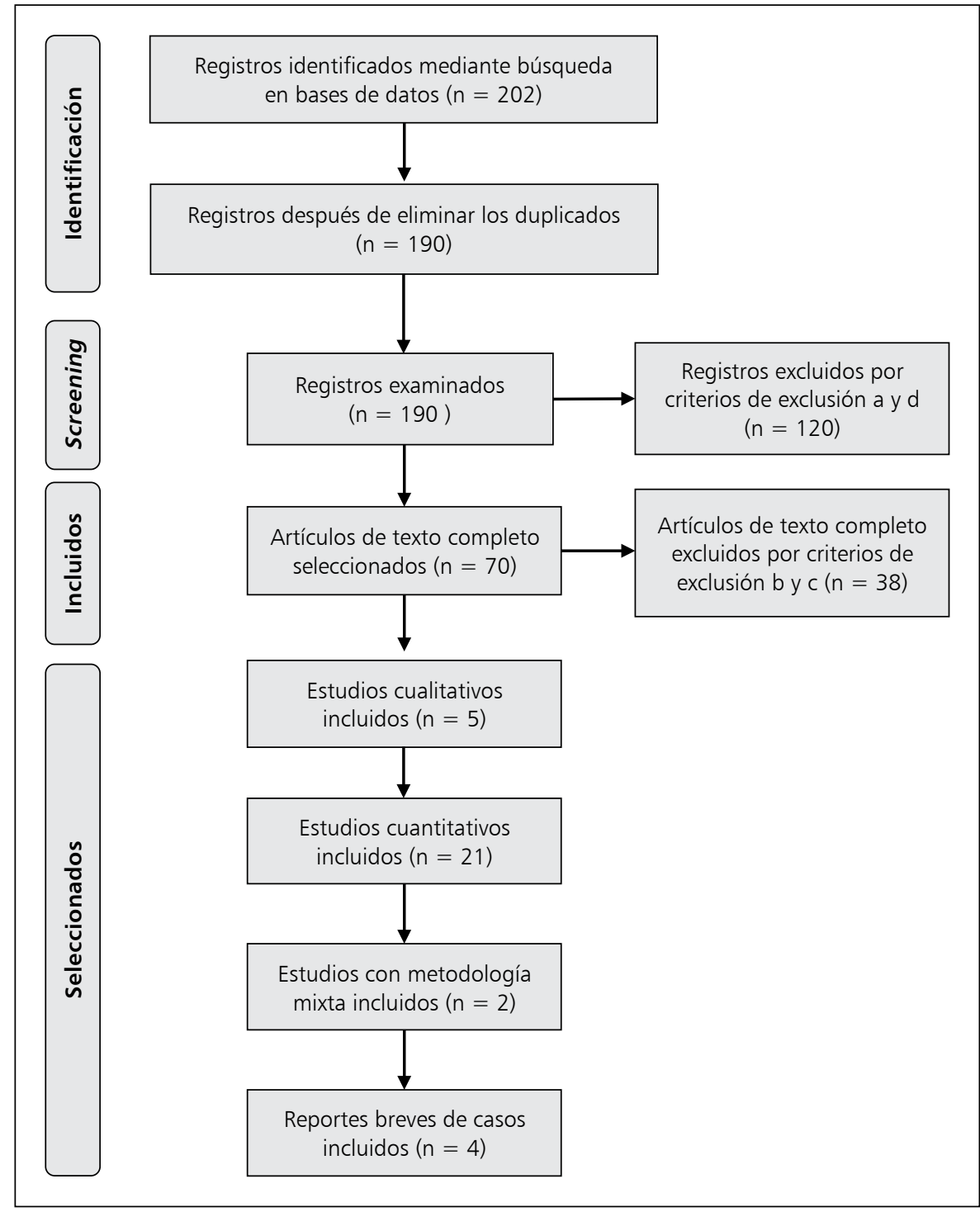

Figura 1. Proceso de búsqueda y selección de los artículos revisados. 


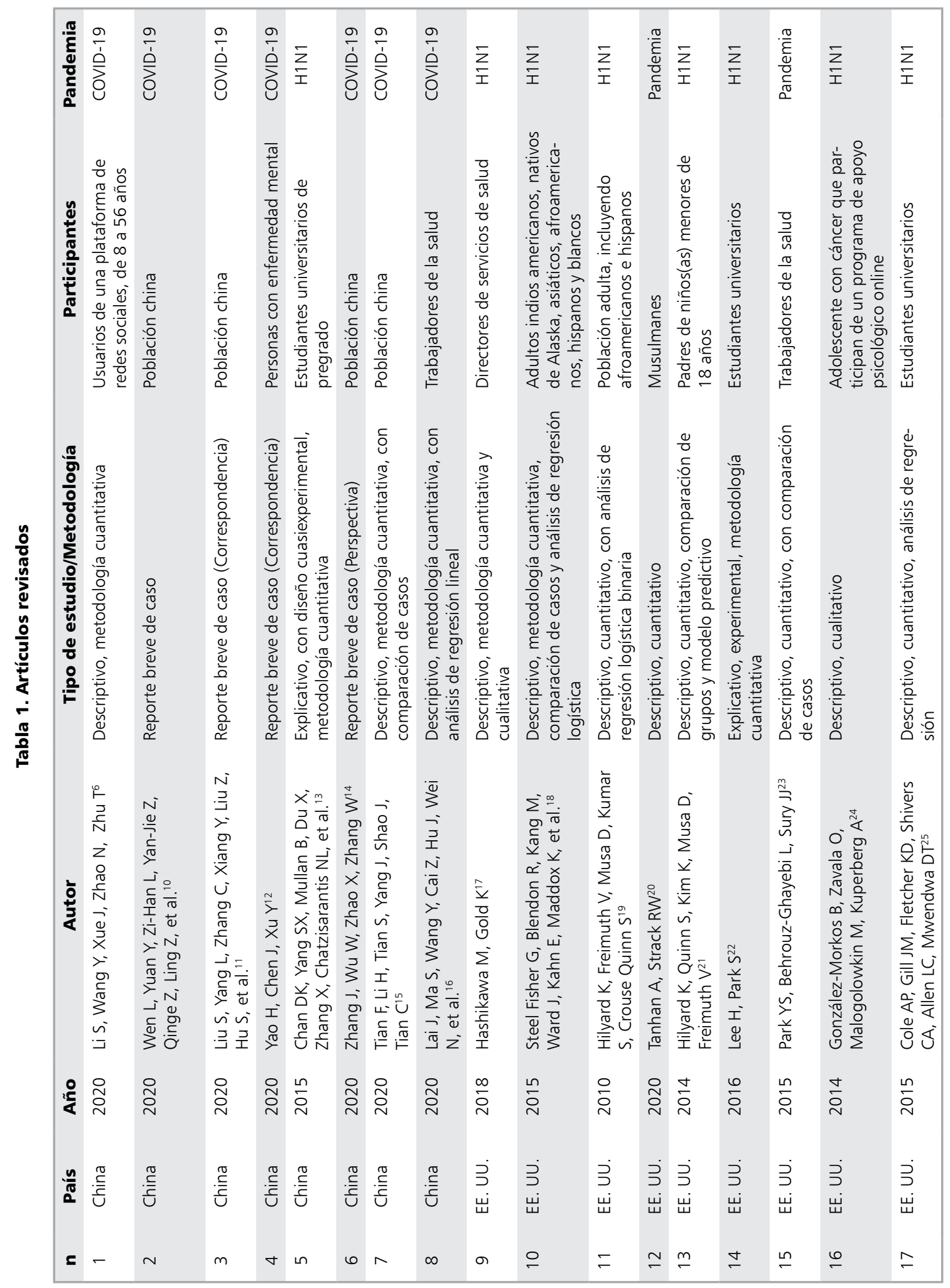




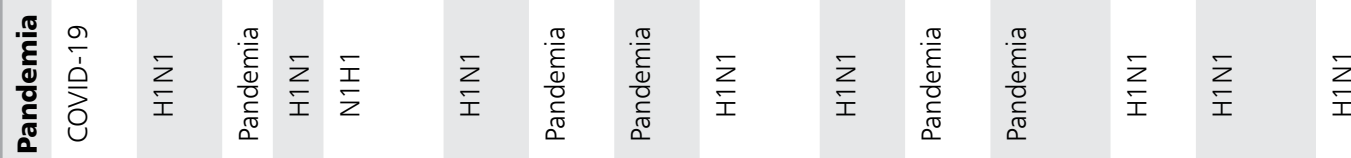

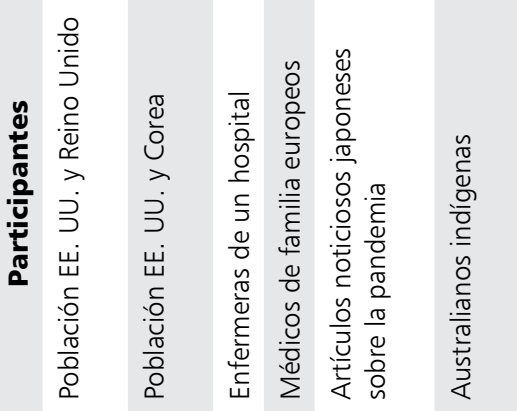

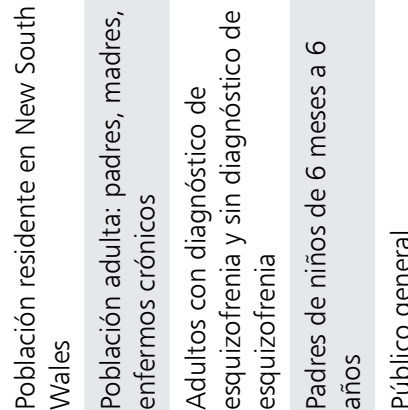

宅 穴

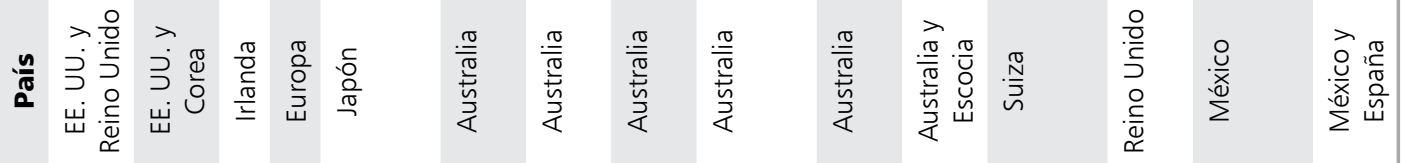

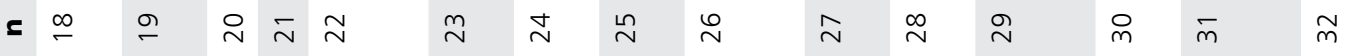




\section{Resultados}

A continuación, se detallan cada una de las categorías de análisis de los artículos.

\section{Impacto psicológico de la pandemia}

Los resultados revelan que las pandemias producen importantes impactos psicológicos. Dos son los factores que impactan psicológicamente en la población: (a) la percepción de más o menos riesgo de la integridad física y psicológica propia y de la comunidad, las organizaciones y los grupos sociales, por la enfermedad pandémica y (b) la alteración del ciclo vital de las personas. Estos afectan negativamente la satisfacción vital ${ }^{6}$, la salud mental ${ }^{6-11}$, los hábitos de vida ${ }^{8}$, el funcionamiento de las organizaciones ${ }^{17-28-23}$ y de los grupos sociales $^{31-12}$. El análisis de los estudios empíricos revisados permitió clasificar el impacto psicológico de una pandemia en las dimensiones: a) emocional; b) cognitiva; c) conductual; d) salud mental; e) organizacional; f) psicosocial.

La Tabla 2 muestra el impacto psicológico en base a estas categorías.

Los estudios revisados también muestran efectos psicológicos específicos de la pandemia, a partir de las características que presentan los grupos sociales y la condición en que se encuentran respecto de la enfermedad. Este impacto puede ir desde una mayor a una menor vulnerabilidad psicológica ${ }^{6}$ : en (a) un nivel primario, se encuentran personas que han sido diagnosticadas con la enfermedad pandémica y bajo la medida de hospitalización por presentar condiciones de salud desfavorables asociadas a esta. También se incluye aquí al personal de salud de primera línea de atención; (b) el nivel secundario, lo integran personas diagnosticadas con la enfermedad pandémica o que se sospecha presentan la enfermedad y, por lo tanto, se encuentran con medidas de aislamiento; (c) en el tercer nivel, se encuentran aquellos que son parte de la red social de las personas de los niveles uno y dos, por ejemplo, los familiares, amigos y grupos de voluntarios que trabajan para mitigarla; (d) el cuarto nivel corresponde a las personas afectadas por las medidas de prevención y control pandémico y que no necesariamente se encuentran contagiadas.

Otros grupos sociales en los que se observa un impacto psicológico particular de la pandemia son la familia, los grupos de menores ingresos económicos, las personas con enfermedad mental y grupos étnicos específicos. Estos dos últimos grupos son indicados como especialmente vulnerables psicológicamente en contexto de la pandemia. Las personas con enfermedad mental, sobre todo quienes se encuentran hospitalizados como medida de rehabilitación de su salud mental, son especialmente vulnerables por el deterioro cognitivo, la baja conciencia del riesgo, el confinamiento en los psiquiátricos, las mayores barreras de acceso a atención médica en caso de contagio, por efecto de la discriminación de este tipo de pacientes, y un mayor impacto de la "epidemia de miedo", que puede desajustar mucho más a una persona que ya presenta una psicopatología de base ${ }^{12}$. Por ejemplo, en Australia ${ }^{34}$ se encontró que las personas con esquizofrenia presentan una menor disposición a vacunarse por la pandemia $H 1 N 1$, dado que perciben un mayor riesgo de que la vacuna produzca reacciones adversas o genere un estado gripal; también que consideran las medidas de aislamiento social menos efectivas. Las minorías étnicas son otro grupo que merece especial atención por los efectos psicológicos de la pandemia. Estos grupos minoritarios pueden presentar un menor acceso a la salud por discriminación y racismo, además de una mayor presencia de enfermedades crónicas ${ }^{31}$. También se ha encontrado que las medidas de cierre de fronteras por pandemia pueden tener a la base el apoyo de la comunidad por motivaciones mixtas, en donde una de las razones de peso es más bien un sentimiento de rechazo a la inmigración más que de seguridad sanitaria ${ }^{19}$.

La Tabla 3 muestra el impacto psicológico por pandemia, en los grupos sociales de los que se tiene reporte y que merecen especial atención.

\section{2. ¿Adherir o no las medidas para la pandemia?}

Los estudios revisados evidencian que ciertas condiciones psicosociales son determinantes en la adherencia o no a las medidas de abordaje ante la pandemia. La adherencia a las medidas de abordaje depende de a) la capacidad para transmitir la información sobre las medidas y la capacidad de la población de recepcionarlas; b) la evaluación de las medidas por parte de la población; c) las características de la relación interpersonal entre quienes entregan las medidas y quienes la reciben; d) los factores psicosociales que favorecen o limitan la adherencia a las distintas medidas. La Tabla 4 presenta una descripción detallada de estos determinantes. 


\section{Tabla 2. Dimensiones de impacto psicológico por una pandemia}

\begin{tabular}{|c|c|c|c|}
\hline Dimensión & Descripción & País & Fuente \\
\hline Emocional & $\begin{array}{l}\text { Disminución de emociones positivas y aumento de emociones ne- } \\
\text { gativas: disminución de la felicidad, del sentimiento de seguridad y } \\
\text { confianza y la satisfacción vital } \\
\text { Aumento de la ansiedad, ira, malestar, inseguridad, miedo, soledad, } \\
\text { desesperación } \\
\text { Miedo colectivo }\end{array}$ & $\begin{array}{l}\text { China } \\
\text { Japón }\end{array}$ & $\begin{array}{l}1-2 \\
22\end{array}$ \\
\hline \multirow[t]{2}{*}{ Conductual } & $\begin{array}{l}\text { Agotamiento físico } \\
\text { Disminución de actividades de ocio }\end{array}$ & China & 1 \\
\hline & $\begin{array}{l}\text { Tendencia a un rechazo a vacunar a los hijos, por parte de los pa- } \\
\text { dres, excepto cuando estos presentan enfermedades de base }\end{array}$ & EE. UU. & 13 \\
\hline \multirow[t]{4}{*}{ Cognitiva } & $\begin{array}{l}\text { Aumento de la desconfianza } \\
\text { Aumento de las creencias religiosa } \\
\text { Aumento de la evaluación del riesgo o negación de este } \\
\text { Incertidumbre } \\
\text { Decepción } \\
\text { Mayor preocupación por la salud y la familia } \\
\text { Mayor frecuencia de pensamientos de muerte }\end{array}$ & China & $1-2$ \\
\hline & $\begin{array}{l}\text { Preocupación por la falta de apoyo en grupos específicos de } \\
\text { inmigrantes }\end{array}$ & EE. UU. & 12 \\
\hline & $\begin{array}{l}\text { Valoración de las medidas de protección en las campañas de salud, } \\
\text { pero baja confianza en su eficacia }\end{array}$ & Australia y Escocia & 28 \\
\hline & Desinformación & $\begin{array}{l}\text { Europa } \\
\text { EE. UU. - Reino Unido }\end{array}$ & $\begin{array}{l}21 \\
18\end{array}$ \\
\hline Salud mental & $\begin{array}{l}\text { Depresión } \\
\text { Estrés } \\
\text { Ansiedad } \\
\text { Trastornos del sueño } \\
\text { Autoagresión } \\
\text { Suicidio } \\
\text { Síntomas obsesivos compulsivos } \\
\text { Dificultad con el duelo }\end{array}$ & China & $2-3-7-8$ \\
\hline \multirow[t]{4}{*}{$\begin{array}{l}\text { Organizacional } \\
\text { de institución } \\
\text { de salud }\end{array}$} & $\begin{array}{l}\text { Mínima existencia de planes en instituciones de salud para abordar } \\
\text { pandemias, asociado a una baja percepción de riesgo. Tendencia al } \\
\text { desconocimiento de las barreras organizacionales para el abordaje } \\
\text { de una pandemia } \\
\text { Confianza en que los trabajadores se mantendrán en sus cargos } \\
\text { durante una pandemia }\end{array}$ & EE. UU. & 9 \\
\hline & $\begin{array}{l}\text { Mínimas condiciones de prevención por confinamiento en institucio- } \\
\text { nes psiquiátricas, en personas con enfermedad mental }\end{array}$ & China & 4 \\
\hline & $\begin{array}{l}\text { En enfermeras estrés laboral, sobrecarga de trabajo y temor a con- } \\
\text { tagiarse ellas y su familia } \\
\text { Compromiso social y sentido de autoeficacia en enfermeras }\end{array}$ & Irlanda & 20 \\
\hline & Presión en los médicos por lograr la inmunización en la población & Europa & 21 \\
\hline \multirow{4}{*}{$\begin{array}{l}\text { Psicosocial y } \\
\text { comunicacional }\end{array}$} & Distorsión de la información en los medios comunicacionales & Europa & 21 \\
\hline & $\begin{array}{l}\text { Influencia de los medios de prensa en la representación social de } \\
\text { la pandemia }\end{array}$ & México-España & 32 \\
\hline & Estigmatización y prejuicio hacia los contagiados & Japón & 22 \\
\hline & $\begin{array}{l}\text { Discriminación en el acceso a programas de salud para la pandemia, } \\
\text { en personas con enfermedad mental }\end{array}$ & China & 4 \\
\hline
\end{tabular}




\section{Tabla 3. Impacto psicológico por la pandemia en distintos grupos sociales}

\begin{tabular}{|c|c|c|c|}
\hline Grupo & Impacto psicológico & País & Fuente \\
\hline \multirow[t]{5}{*}{ Familia } & $\begin{array}{l}\text { Dificultad con el duelo cuando un integrante fallece por diagnóstico de enferme- } \\
\text { dad pandémica }\end{array}$ & China & 2 \\
\hline & $\begin{array}{l}\text { Necesidad de información para proteger a los hijos de la pandemia: qué hacer en } \\
\text { caso de infección y cómo prevenirla }\end{array}$ & Australia & 27 \\
\hline & Dudas sobre la medida de vacunar a sus hijos(as) & EE. UU. & 13 \\
\hline & Mayor preocupación por la familia & China & 1 \\
\hline & Preocupación por la salud física de miembros que trabajan en salud & Irlanda & 20 \\
\hline \multirow[t]{2}{*}{$\begin{array}{l}\text { Personas con } \\
\text { enfermedad } \\
\text { mental }\end{array}$} & $\begin{array}{l}\text { El deterioro cognitivo, la poca conciencia del riesgo, las condiciones confinadas } \\
\text { en salas psiquiátricas, las mayores barreras de acceso a la salud por contagio y a } \\
\text { tratamiento psicológico, conllevan a una mayor afectación psicológica }\end{array}$ & China & 4 \\
\hline & $\begin{array}{l}\text { Menor disposición a vacunarse ante la pandemia } \\
\text { Mayor percepción de riesgo de vacunarse, respecto de personas sin esquizofrenia, } \\
\text { por las reacciones adversas para la salud y la posible generación de un estado gripal } \\
\text { Menor confianza en las medidas de aislamiento social }\end{array}$ & Australia & 26 \\
\hline \multirow[t]{5}{*}{$\begin{array}{l}\text { Trabajadores } \\
\text { de la salud } \\
\text { (enfermeras y } \\
\text { médicos) }\end{array}$} & $\begin{array}{l}\text { En una alta proporción, preocupación por el mayor riesgo de exposición a la } \\
\text { infección } \\
\text { En una proporción media, miedo a contraer la infección } \\
\text { En una proporción media, desconfianza en los cuidados y protección del empleador } \\
\text { en caso de contagio } \\
\text { Alta proporción de temor a que sus familiares y compañeros de trabajo se con- } \\
\text { tagien } \\
\text { Mayores niveles de estrés laboral y conflictos en el trabajo } \\
\text { Autoeficacia profesional para abordar la pandemia }\end{array}$ & Irlanda & 20 \\
\hline & $\begin{array}{l}\text { Inconformidad con las campañas preventivas del gobierno, grupos de profesionales } \\
\text { y organizaciones por una falta de coordinación y de información oportuna } \\
\text { Presión psicológica por las exigencias de las autoridades de inmunización de la } \\
\text { población y por las demandas de protección de los pacientes }\end{array}$ & Europa & 21 \\
\hline & Síntomas de ansiedad, depresión, insomnio y angustia & China & 8 \\
\hline & $\begin{array}{l}\text { Carga de trabajo excesiva, aislamiento y discriminación } \\
\text { Alta vulnerabilidad a experimentar agotamiento físico, miedo, alteraciones emocio- } \\
\text { nales y problemas de sueño, síntomas depresivos, ansiedad y alteración del sueño }\end{array}$ & China & $2-7$ \\
\hline & Cambios en la disposición a trabajar durante una pandemia & EE. UU. & 15 \\
\hline \multirow[t]{5}{*}{ Grupos étnicos } & $\begin{array}{l}\text { Mayor receptividad a medidas preventivas y de protección de la salud para evitar } \\
\text { el contagio } \\
\text { Mayor percepción de riesgo de ser infectados o de que sus familias se infecten } \\
\text { Mayor percepción de riesgo de contagio }\end{array}$ & EE. UU. & 10 \\
\hline & Sentimiento de rechazo hacia los inmigrantes justificado con la pandemia & EE. UU. & 11 \\
\hline & Preocupación por la falta de apoyo & & 12 \\
\hline & Discriminación y mayores barreras de acceso a la salud & Australia & 23 \\
\hline & $\begin{array}{l}\text { Exclusión social manifestada en un exigente perfil de inmigrante para el acceso a } \\
\text { un país, que incluye en entre otras condiciones, el estar saludable } \\
\text { Exclusión social manifestada en el deseo de disminuir el número de inmigrantes } \\
\text { de un país }\end{array}$ & Suiza & 2 \\
\hline \multirow[t]{2}{*}{$\begin{array}{l}\text { Grupos de } \\
\text { menores } \\
\text { ingresos } \\
\text { económicos }\end{array}$} & $\begin{array}{l}\text { Población de menores ingresos y de menor educación son menos propensos a } \\
\text { apoyar medidas de racionamiento de la vacuna priorizadas por población } \\
\text { Medidas de cuarentena vividas con mayor dificultad en sectores de menores ingre- } \\
\text { sos, por falta de recursos y exigencias económicas-laborales }\end{array}$ & EE. UU. & 11 \\
\hline & $\begin{array}{l}\text { Menor identificación de redes de apoyo familiares y sociales } \\
\text { Mayor adherencia a creencias religiosas para obtener soporte emocional }\end{array}$ & Australia & 24 \\
\hline
\end{tabular}




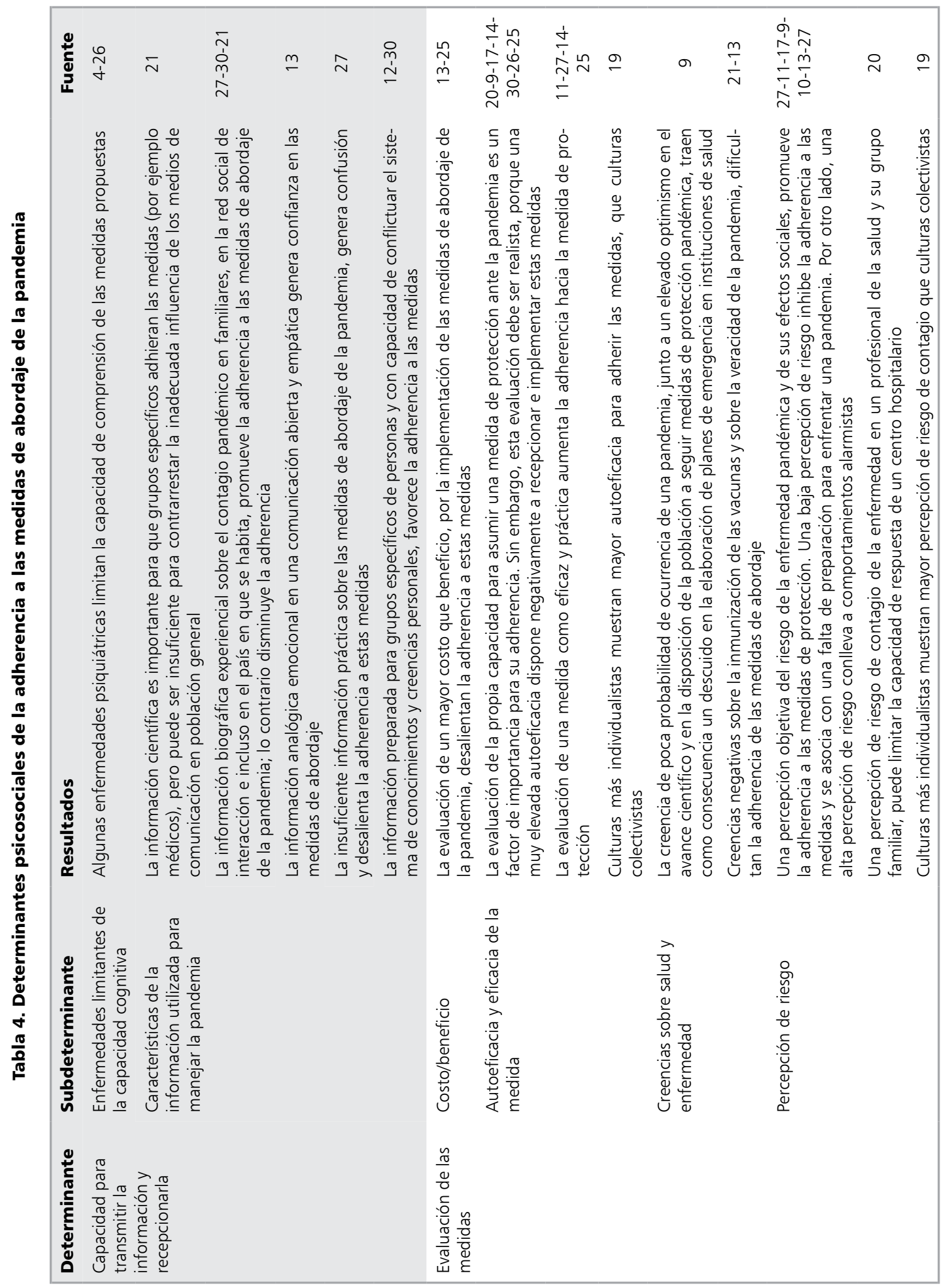




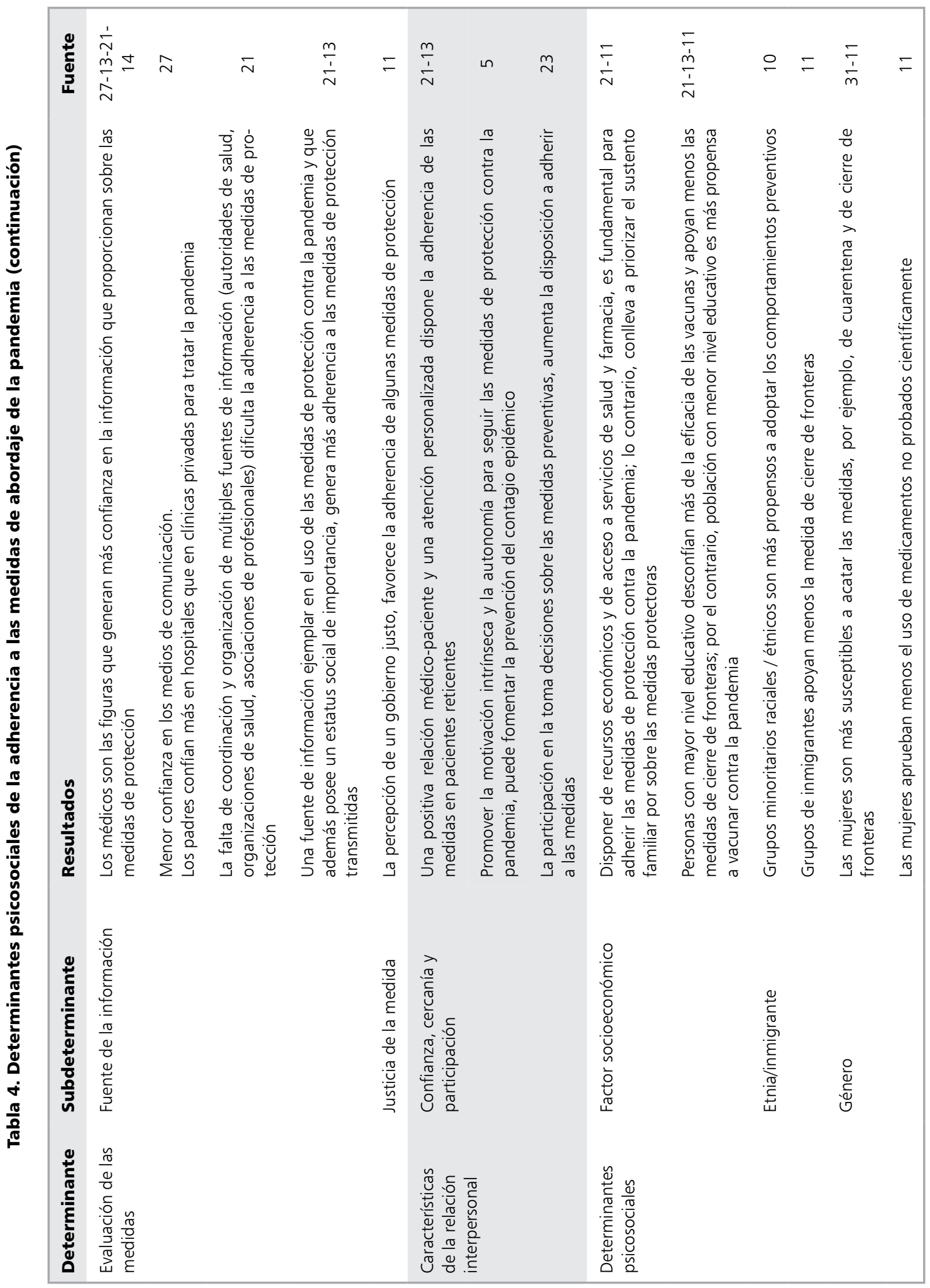




\section{Consideraciones psicosociales para el abordaje de la pandemia}

Del análisis de los estudios revisados, se desprenden una serie de consideraciones psicosociales a la hora de planificar las medidas de abordaje pandémico.

La Tabla 5 muestra seis dimensiones claves: (a) la forma de informar a la población; (b) las características del apoyo psicológico; (c) las acciones educativas sobre la pandemia o relacionadas con ésta; (d) la capacitación requerida para grupos específicos, (e) la organización de los apoyos; (f) y la evaluación y monitoreo de la salud mental poblacional.

\section{Metodología de los estudios revisados}

El $65,6 \%$ de los estudios revisados utiliza metodología cuantitativa, $15,6 \%$ metodología cualitativa, mientras que $6,3 \%$ utilizó un método mixto. El 50\% son estudios descriptivos, 18,8\% incluye comparación de grupos. Mientras que $31,3 \%$ son estudios que buscaron explicar, de los cuales 1 utiliza un diseño cuasiexperimental, 1 un diseño experimental y la mayoría establece relaciones causales en base a análisis de regresión o ecuaciones estructurales.

\section{Discusión y Conclusiones}

El propósito de este trabajo fue sistematizar el conocimiento científico que se tiene a la fecha sobre el comportamiento psicológico en situación de pandemia, a partir de los estudios empíricos y de reportes breves de caso, en el contexto de las pandemias declaradas durante el siglo XXI: la influenza H1N1 y el COVID-19.

Los estudios revisados develan un impacto psicológico multidimensional importante a nivel de personas, grupos y organizaciones ${ }^{4-6}$. Las pandemias H1N1 y de COVID-19 han producido cambios drásticos en el comportamiento psicosocial de la población, pudiendo disminuir la satisfacción vital e inducir estados emocionales de temor, inseguridad e incertidumbre, además de trastornos psicológicos en las situaciones más graves. La forma de convivencia de los grupos sociales y el funcionamiento de las organizaciones se ha visto desafiada y ha producido problemas importantes que pueden conllevar incluso a la ruptura de su funcionamiento y adaptación ante estos ${ }^{2}$.
Dos son los factores que impactan psicológicamente en la población: el riesgo de contraer la enfermedad pandémica ${ }^{5}$ y las características del abordaje de afrontamiento tomadas por las personas, los grupos sociales y las autoridades de gobierno que, en términos generales, rompen con las rutinas de reproducción cotidiana ${ }^{41-42}$.

Este impacto se da de manera diferenciada en determinados grupos sociales que, por sus características, pueden encontrarse en una condición de mayor vulnerabilidad psicosocial ante la emergencia pandémica ${ }^{34}$. Los estudios revisados no agotan los grupos sociales necesarios de investigar, por lo que se requiere redoblar los esfuerzos para dar cuenta del comportamiento psicosocial ante una pandemia. Por ejemplo, las características de las localidades que destacan por un abordaje y control adecuado de la pandemia; de los adultos mayores por ser una de las poblaciones más vulnerables a la infección; la población educativa, tales como profesores y estudiantes, dada la alta presión por retornar a la "normalidad" o mantener su funcionamiento; de los pacientes con enfermedades crónicas. También es necesario aumentar el cuerpo de conocimientos sobre el personal de salud, dado que es un grupo altamente expuesto, cuya labor es decisiva en el manejo de la enfermedad pandémica $^{28}$. Sobre todo, se requiere diseñar, implementar y validar intervenciones psicológicas efectivas para estos grupos ${ }^{43}$, más aun en contexto de emergencia pandémica ${ }^{14-44}$.

Es urgente continuar avanzando con la realización de más estudios que permitan describir mejor el comportamiento psicológico pandémico $y$, sobre todo, con estudios explicativos. Por ejemplo, estudios longitudinales y que identifiquen las variables psicosociales que son predictores de la adherencia de la población a las medidas de enfrentamiento ante la pandemia. También se requiere de más investigaciones cualitativas, dada la baja proporción encontrada en los estudios revisados y la necesidad de comprender mejor las experiencias subjetivas frente a la pandemia, a fin de ampliar el conocimiento al respecto y desde allí, mejorar las estrategias de intervención psicológica preventivas y de contención. El desafío queda puesto en las características de estos diseños, dado que en situación de pandemia el acceso a la población es una dificultad importante. En varios trabajos revisados el levantamiento de información online permitió el acceso a los participantes 
Tabla 5. Consideraciones psicosociales para el abordaje de la pandemia

\begin{tabular}{|c|c|c|c|}
\hline Dimensión & Temática & País & Fuente \\
\hline \multirow{6}{*}{$\begin{array}{l}\text { Informar a la } \\
\text { población }\end{array}$} & \multirow{4}{*}{$\begin{array}{l}\text { Proporcionar información precisa y oportuna sobre la evolución de la pan- } \\
\text { demia, los avances en su manejo, los apoyos entregados y el acceso a estos }\end{array}$} & China & 1 \\
\hline & & Japón & 22 \\
\hline & & EE. UU. & 11 \\
\hline & & Europa & 21 \\
\hline & $\begin{array}{l}\text { Proporcionar información precisa y clara sobre los tratamientos de salud y } \\
\text { cómo acceder a ellos }\end{array}$ & China & $1-3$ \\
\hline & $\begin{array}{l}\text { Planificar campañas informativas que generen un conflicto cognitivo en la } \\
\text { población reticente a seguir las medidas preventivas }\end{array}$ & $\begin{array}{l}\text { Reino } \\
\text { Unido }\end{array}$ & 30 \\
\hline \multirow{7}{*}{$\begin{array}{l}\text { Apoyar } \\
\text { psicológicamente } \\
\text { a la población }\end{array}$} & \multirow{2}{*}{$\begin{array}{l}\text { Proporcionar apoyo psicológico online para la salud mental de la población. } \\
\text { La intervención en crisis debe centrarse en el miedo, la adaptación y resiliencia } \\
\text { psicológica }\end{array}$} & China & $1-3-6$ \\
\hline & & EE. UU. & 15 \\
\hline & $\begin{array}{l}\text { Mantener los apoyos psicológicos de grupos que presentan enfermedades } \\
\text { médicas que afectan su salud mental o presentan trastornos psicológicos, en } \\
\text { base a una modalidad online }\end{array}$ & EE. UU. & 16 \\
\hline & $\begin{array}{l}\text { Proporcionar atención psicológica para el cuidado de la salud mental del } \\
\text { personal de salud }\end{array}$ & $\begin{array}{l}\text { China } \\
\text { Irlanda }\end{array}$ & $\begin{array}{c}2-6-3 \\
20\end{array}$ \\
\hline & $\begin{array}{l}\text { Disponer orientaciones a la familia, para superar el duelo por fallecimiento de } \\
\text { familiares por la pandemia }\end{array}$ & China & 2 \\
\hline & $\begin{array}{l}\text { Proporcionar asesoramiento psicológico organizacional a organismos que se } \\
\text { encargan de la pandemia }\end{array}$ & China & 2 \\
\hline & Mantener las psicoterapias para personas con trastornos mentales & China & $3-4$ \\
\hline \multirow{8}{*}{$\begin{array}{l}\text { Educar a la } \\
\text { población } \\
\text { considerando } \\
\text { grupos específicos }\end{array}$} & \multirow[t]{2}{*}{ Educar sobre la pandemia y la forma de afrontarla } & China & 2 \\
\hline & & Australia & 27 \\
\hline & $\begin{array}{l}\text { Educar sobre el impacto psicológico pandémico, las estrategias de afronta- } \\
\text { miento y las capacidades de adaptación }\end{array}$ & China & $2-3-6$ \\
\hline & Educar en promoción de salud mental & China & $2-6$ \\
\hline & Educar sobre las fuentes confiables de información sobre la pandemia & Australia & 27 \\
\hline & \multirow{2}{*}{$\begin{array}{l}\text { Disponer campañas educativas preventivas que fomenten la motivación in- } \\
\text { trínseca, vinculen el modelado y la experiencia biográfica de personajes con } \\
\text { autoridad o célebres }\end{array}$} & China & 3 \\
\hline & & EE. UU. & 13 \\
\hline & $\begin{array}{l}\text { Asegurar la participación de comunidades étnicas en la planificación del abor- } \\
\text { daje de la pandemia, para fomentar la autodeterminación y responsabilidad } \\
\text { por las medidas preventivas }\end{array}$ & Australia & 23 \\
\hline \multirow{7}{*}{$\begin{array}{l}\text { Capacitar al } \\
\text { personal de salud } \\
\text { y de salud mental }\end{array}$} & \multirow{7}{*}{$\begin{array}{l}\text { Conocimiento de la pandemia en personal de salud mental } \\
\text { Intervención psicológica breve y/o primeros auxilios psicológicos ante la crisis } \\
\text { pandémica } \\
\text { Salud mental en pandemia } \\
\text { Apoyo psicológico a pacientes con un severo deterioro de su salud mental } \\
\text { Planes para abordar la emergencia en instituciones sanitarias } \\
\text { Distribución de roles, funciones, horarios y apoyos al personal de salud durante } \\
\text { la emergencia }\end{array}$} & China & 1 \\
\hline & & China & $2-3$ \\
\hline & & China & 2 \\
\hline & & China & 2 \\
\hline & & EE. UU. & 9 \\
\hline & & Irlanda & 20 \\
\hline & & EE. UU. & 15 \\
\hline \multirow{4}{*}{$\begin{array}{l}\text { Organizar los } \\
\text { apoyos }\end{array}$} & \multirow{4}{*}{$\begin{array}{l}\text { Lograr la coordinación de científicos, profesionales de la salud, gobiernos } \\
\text { locales y organismos gubernamentales para informar, educar y apoyar a la } \\
\text { población } \\
\text { Asegurar apoyos legales y económicos a la población, para que pueda seguir } \\
\text { las medidas de prevención, sobre todo en la población de menores ingresos } \\
\text { Asegurar la recreación saludable de la familia durante una cuarentena }\end{array}$} & China & 2 \\
\hline & & Europa & 21 \\
\hline & & EE. UU. & $11-17$ \\
\hline & & China & 1 \\
\hline \multirow{2}{*}{$\begin{array}{l}\text { Evaluar la salud } \\
\text { mental de la } \\
\text { población }\end{array}$} & Elaborar e implementar encuestas de salud mental & China & $3-6$ \\
\hline & $\begin{array}{l}\text { Elaborar mecanismos para identificar psicopatologías y riesgo de suicidio } \\
\text { poblacional }\end{array}$ & China & 3 \\
\hline
\end{tabular}


de los estudios. Sin embargo, es preciso discutir e investigar lo adecuado de estos medios y refinar los diseños a partir de los hallazgos de estos estudios.

No obstante, de la crítica anterior, la respuesta psicológica ante la pandemia que se desprende de los estudios revisados, permite sacar lecciones e informar para la toma decisiones en la forma de abordaje de las pandemias. El desafío que viene es evaluar el resultado de las medidas y acciones tomadas en el contexto de las pandemias ocurridas durante el siglo XXI. Aquí, nuevamente la ciencia debe aportar evidencia que devele la eficiencia del abordaje psicológico de la pandemia y oriente sobre cómo los países pueden desarrollar capacidades de afrontamiento y resiliencia a partir de la experiencia adquirida ante estos riesgos y promover un fortalecimiento psicosocial y de la salud mental de la población post emergencia pandémica ${ }^{14-44}$. Estos trabajos pueden ser una pieza clave para mejorar el abordaje psicosocial de las pandemias ${ }^{45}$. Sumado a lo anterior, un tema pendiente respecto de la respuesta psicológica ante la pandemia por COVID-19 es la percepción y disposición ante la vacuna para esta enfermedad. Es probable que la percepción de la población presente variaciones respecto de la vacuna para la influenza N1H1, situación que futuros estudios deberían develar.

Una fortaleza de la presente revisión es haber establecido categorías y dimensiones que permitieron analizar los resultados de los estudios incluidos en ella, las que podrían ser usadas para el análisis de investigaciones en desarrollo y futuras que aún no han sido publicadas. Una limitación de esta revisión es la diversidad de la calidad metodológica de los artículos, por ejemplo, en los tamaños muestrales, los instrumentos de recolección de información utilizados y su aplicación bajo un contexto pandémico, lo que influyó en las posibilidades de análisis y, por tanto, en la elaboración de conclusiones. Uno de los grandes desafíos de la investigación científica del comportamiento psicológico pandémico es mejorar los diseños metodológicos a utilizar durante la fase de emergencia.

Otra posible debilidad de este trabajo es el hecho de que no se incluyó literatura gris, dada la dificultad de acceso a esta información, la cautela que se requiere respecto a la calidad de ella y lo inestable que resulta su almacenaje en repositorios o bases de datos.
Finalmente, es necesario convocar a la comunidad científica al despliegue de todos sus esfuerzos para proporcionar a la población una mayor comprensión del comportamiento de las personas en situación de pandemia. El comportamiento psicológico pandémico es más que nunca una dimensión que debe orientar, tanto las medidas políticas de control $^{46}$ como las intervenciones psicológicas y en salud en general efectivas para el manejo de las pandemias.

\section{Referencias}

1. World Health Organization. Virtual Press Conference on COVID-19 - 11 March 2020. 11 march 2020). Disponible en: https://webcache.googleusercontent. com/search?q=cache:ITPUJt2LHBQJ:https://www. who.int/docs/default-source/coronaviruse/transcripts/ who-audio-emergencies-coronavirus-press-conference [Consultado el 25 de abril de 2020].

2. Kamradt-Scott A. The Politics of Pandemic Influenza Preparedness. In: Colin McInnes, Kelley Lee, Jeremy Youde, Editors, The Oxford Handbook of Global Health Politics. Oxford, Oxford University Press: Oxford handbooks Online; 2020. p. 1-23. Disponible en https://www.oxfordhandbooks.com/ view/10.1093/oxfordhb/9780190456818.001.0001/ oxfordhb-9780190456818-e-3 [Consultado el 20 de abril de 2020].

3. Jiménez JP. (2020, abril). Entrevista con Juan Pablo Jiménez. Recuperada de https://tv.emol.com/ detail/20200428172657361/en-vivo-juan-pablo-jimenez-aborda-la-incertidumbre-por-covid

4. Sadati A, Lankarani M, Lankarani K. Risk Society, Global Vulnerability and Fragile Resilience; Sociological View on the Coronavirus Outbreak. Shiraz E-Med J. (in press). Disponible en: http://emedicalj.com/articles/102263.html [Consultado en 25 de julio de 2020].

5. Zakrzewska M, Liuzza M, Lindholm T, Blomkvist A, Larsson A, Olofsson J. An Overprotective Nose? Implicit Bias Is Positively Related to Individual Differences in Body Odor Disgust Sensitivity. Frontiers in Psychology 2020; 11 (301): 1-11. Disponible en: https://www. frontiersin.org/articles/10.3389/fpsyg.2020.00301/full [Consultado el 20 de abril de 2020].

6. Li S, Wang Y, Xue J, Zhao N, Zhu T. The Impact of COVID-19 Epidemic Declaration on Psychological Consequences: A Study on Active Weibo Users. Int. J. Environ. Res. Public Health 2020; 17 (6): 1-9. Disponible en: https://www.mdpi.com/1660-4601/17/6/2032 [Consultado el 25 de marzo de 2020]. 
7. Philip R. COVID-19 and mental health: A review of the existing literatura. Asian Journal of Psychiatry 2020; 52: 1-5. Disponible en: https://www.ncbi.nlm.nih.gov/ pmc/articles/PMC7151415/ [Consultado el 25 de abril de 2020].

8. Taylor S. The Psychology of Pandemics: Preparing for the Next Global Outbreak of Infectious Disease. Cambridge, United Kingdom: Cambridge Scholars Publishing 2019.

9. Reyes BH. Artículos de Revisión. Rev Med Chile [Internet]. 2020 Ene [citado el 29 de abril de 2020]; 148 (1): 103-8. Disponible en: https://scielo.conicyt.cl/ scielo.php?script=sci_arttext\&pid=S0034-98872020000 100103\&lng=es.

10. Wen L, Yuan Y, Zi-Han L, Yan-Jie Z, Qinge Z, Ling Z, et al. Progression of Mental Health Services during the COVID-19 Outbreak in China. Int J Biol Sci. 2020; 16 (10): 1732-8. Disponible en: https://www.ncbi.nlm.nih. gov/pmc/articles/PMC7098037/citedby/ [Consultado el 04 de abril de 2020].

11. Liu S, Yang L, Zhang C, Xiang Y, Liu Z, Hu S, et al. Online mental health services in China during the COVID-19 outbreak. The Lancet Psychiatry 2020; 7: e17-e18. Disponible en: https://www.thelancet.com/ journals/lanpsy/article/PIIS2215-0366(20)30077-8/ fulltext [Consultado el 15 de abril de 2020].

12. Yao H, Chen J, Xu Y. Patients with mental health disorders in the COVID-19 epidemic. The Lancet Psychiatry 2020; 7 (4): e21. Disponible en: https://www.thelancet. com/journals/lanpsy/article/PIIS2215-0366(20)30090-0/ fulltext [Consultado el 15 de abril de 2020].

13. Chan DK, Yang SX, Mullan B, Du X, Zhang X, Chatzisarantis NL, et al. Preventing the spread of H1N1 influenza infection during a pandemic: autonomy-supportive advice versus controlling instruction. J Behav Med. 2015; 38 (3): 416-26. Disponible en: https://www. ncbi.nlm.nih.gov/pubmed/25542273 [Consultado el 15 de abril de 2020].

14. Zhang J, Wu W, Zhao X, Zhang W. Recommended psychological crisis intervention response to the 2019 novel coronavirus pneumonia outbreak in China: a model of West China Hospital. Precision Clinical Medicine; 3 (1): 3-8. Disponible en: https://doi.org/10.1093/pcmedi/ pbaa006 [Consultado el 15 de abril de 2020].

15. Tian F, Li H, Tian S, Yang J, Shao J, Tian C. Psychological symptoms of ordinary Chinese citizens based on SCL-90 during the level I emergency response to COVID-19. Psychiatry Res. [Epub ahead of print]; 288. Disponible en: https://www.ncbi.nlm.nih.gov/pubmed/32302816 [Consultado el 25 de abril de 2020].

16. Lai J, Ma S, Wang Y, Cai Z, Hu J, Wei N, et al. Factors
Associated With Mental Health Outcomes Among Health Care Workers Exposed to Coronavirus Disease 2019. JAMA Network Open 2020; 3 (3): 1-12. Disponible en: https://es.jamanetwork.com/journals/jamanetworkopen/fullarticle/2763229 [Consultado el 25 de abril de 2020].

17. Hashikawa M, Gold KJ. Disaster Preparedness in Primary Care: Ready or Not? Disaster Med Public Health Prep. 2018; 12 (5): 644-8. Disponible en: https://www. ncbi.nlm.nih.gov/pubmed/29362006 [Consultado el 25 de abril de 2020].

18. SteelFisher G, Blendon R, Kang M, Ward J, Kahn E, Maddox K, et al. Adoption of preventive behaviors in response to the $2009 \mathrm{H} 1 \mathrm{~N} 1$ influenza pandemic: a multiethnic perspective. Influenza Other Respir Viruses. 2015; 9 (3): 131-42. Disponible en: https://www.ncbi. nlm.nih.gov/pmc/articles/PMC4415697/ [Consultado el 25 de abril de 2020].

19. Hilyard K, Freimuth V, Musa D, Kumar S, Crouse Quinn S. The vagaries of public support for government actions in case of a pandemic. Health Aff (Millwood) 2010; 29 (12): 2294-301. Disponible en: https://www. ncbi.nlm.nih.gov/pmc/articles/PMC3445335/ [Consultado el 25 de abril de 2020].

20. Tanhan A, Strack RW. Online photovoice to explore and advocate for Muslim biopsychosocial spiritual wellbeing and issues: Ecological systems theory and ally development. Curr Psychol 2020 [online]. Disponible en: https://link.springer.com/article/10.1007\%2Fs12144-020-00692-6\#citeas [Consultado el 20 de abril de 2020].

21. Hilyard K, Quinn S, Kim K, Musa D, Freimuth V. Determinants of Parental Acceptance of the H1N1 Vaccine. Health Educ Behav 2014; 41 (3): 307-14. Disponible en: https://www.ncbi.nlm.nih.gov/pmc/articles/ PMC4643946/ [Consultado el 20 de abril de 2020].

22. Lee H, Park SA. Third-Person Effect and Pandemic Flu: The Role of Severity, Self-Efficacy Method Mentions, and Message Source. J Health Commun 2016; 21 (12): 1244-50. Disponible en: https://www.ncbi.nlm.nih.gov/ pubmed/27858585 [Consultado el 20 de abril de 2020].

23. Park YS, Behrouz-Ghayebi L, Sury, JJ. Do shared barriers when reporting to work during an influenza pandemic influence hospital workers' willingness to work? A multilevel framework. Disaster Med Public Health Prep 2015; 9 (2): 175-85. Disponible en: https://www. ncbi.nlm.nih.gov/pubmed/25882124 [Consultado el 20 de abril de 2020].

24. González-Morkos B, Zavala O, Malogolowkin M, Kuperberg A. The teen impact experience: a webcast pilot project for teens with cancer and blood diseases. 
J Pediatr Oncol Nurs 2014; 31 (5): 272-6. Disponible en: https://www.ncbi.nlm.nih.gov/pubmed/24913969 [Consultado el 22 de abril de 2020].

25. Cole AP, Gill JM, Fletcher KD, Shivers CA, Allen LC, Mwendwa DT. Understanding African American college students' H1N1 vaccination decisions. Health Psychol 2015; 34 (12): 1185-90. Disponible en: https://www. ncbi.nlm.nih.gov/pubmed/26148188 [Consultado el 22 de abril de 2020].

26. Geldsetzer P. Use of Rapid Online Surveys to Assess People's Perceptions During Infectious Disease Outbreaks: A Cross-sectional Survey on COVID-19. J Med Internet Res 2020; 22 (4): e18790. Disponible en: https:// www.ncbi.nlm.nih.gov/pubmed/32240094 [Consultado el 22 de abril de 2020].

27. Cho HC, Lee JS. The influence of self efficacy, subjective norms, and risk perception on behavioral intentions related to the H1N1 flu pandemic: A comparison between Korea and the US. Asian Journal of Social Psychology 2015; 18 (4): 311-24. Disponible en: https:// onlinelibrary.wiley.com/doi/abs/10.1111/ajsp.12104 [Consultado el 22 de abril de 2020].

28. McMullan C, Brown GD, O'Sullivan D. Preparing to respond: Irish nurses' perceptions of preparedness for an influenza pandemic. Int Emerg Nurs 2016; 26: 3-7. Disponible en: https://www.ncbi.nlm.nih.gov/pubmed/26597971 [Consultado el 22 de abril de 2020].

29. Balogh S, Papp R, Busa C, Csikos A. Role of health care professionals in preventative activities and vaccination programs during outbreaks. Acta Microbiologica et Immunologica Hungarica 2016; 63 (4): 365-72. Disponible en: https://akjournals.com/view/ journals/030/63/4/article-p365.xml [Consultado el 22 de abril de 2020].

30. Shigemura J, Harada N, Tanichi M, Nagamine M, Shimizu K, Katsuda Y, et al. Rumor-Related and Exclusive Behavior Coverage in Internet News Reports Following the 2009 H1N1 Influenza Outbreak in Japan. Disaster Med Public Health Prep 2015; 9 (4): 459-63. Disponible en: https://www.ncbi.nlm.nih.gov/pubmed/26186580 [Consultado el 22 de abril de 2020].

31. Miller A, Massey PD, Judd J, Kelly J, Durrheim DN, Clough AR, et al. Using a participatory action research framework to listen to Aboriginal and Torres Strait Islander people in Australia about pandemic influenza. Rural Remote Health 2015; 15 (3): 2923. Disponible en: https://www.ncbi.nlm.nih.gov/pubmed/26223560 [Consultado el 22 de abril de 2020].

32. Taylor M, Barr M, Stevens G, Bryson-Taylor D, Agho $\mathrm{K}$, Jacobs J, et al. Psychosocial stress and strategies for managing adversity: measuring population resilience in
New South Wales. Australia. Popul Health Metrics 2010; 8 (28): 2-9. Disponible en: https://doi.org/10.1186/14787954-8-28 [Consultado el 22 de abril de 2020].

33. Flowers P, Davis M, Lohm D, Waller E, Stephenson N. Understanding pandemic influenza behaviour: An exploratory biopsychosocial study. J Health Psychol 2016; 21 (5): 759-69. Disponible en: https://www.ncbi.nlm. nih.gov/pubmed/24957318 [Consultado el 22 de abril de 2020].

34. Maguire PA, Reay RE, Looi JC. Nothing to sneeze at-uptake of protective measures against an influenza pandemic by people with schizophrenia: willingness and perceived barriers. Australas Psychiatry. 2019; 27 (2): 171-8. Disponible en: https://www.ncbi.nlm.nih.gov/ pubmed/30501496 [Consultado el 22 de abril de 2020].

35. King CL, Chow MYK, Wiley KE, Leask J. Much ado about flu: A mixed methods study of parental perceptions, trust and information seeking in a pandemic. Influenza Other Respir Viruses 2018; 12 (4): 514-21. Disponible en: https://www.ncbi.nlm.nih.gov/pubmed/29437291 [Consultado el 20 de marzo de 2020].

36. Lohm D, Davis M, Flowers P, Stephenson N. 'Fuzzy' virus: indeterminate influenza biology, diagnosis and surveillance in the risk ontologies of the general public in time of pandemics. Health, Risk and Society 2015; 17 (2): 115-31. Disponible en: http://eprints.gla. ac.uk/178353/ [Consultado el 20 de abril de 2020].

37. Green E, Krings F, Staerklé C, Bangerter A, Clémence A, Wagner-Egger $\mathrm{P}$, et al. Keeping the vermin out: Perceived disease threat and ideological orientations as predictors of exclusionary immigration attitudes. Journal of Community \& Applied Social Psychology 2010; 20 (4): 299-316. Disponible en: https://onlinelibrary.wiley. com/doi/abs/10.1002/casp.1037 [Consultado el 20 de abril de 2020].

38. Rubin GJ, Finn Y, Potts HW, Michie S. Who is sceptical about emerging public health threats? Results from 39 national surveys in the United Kingdom. Public Health 2015; 129 (12): 1553-62. Disponible en: https://www. ncbi.nlm.nih.gov/pmc/articles/PMC4684148/ [Consultado el 20 de marzo de 2020].

39. Carro E, Hernández A. Percepción de riesgo y prevención en un contexto de emergencia sanitaria caso influenza A (H1N1), 2009. Revista de Psicología y Ciencias del Comportamiento de la U.A.C.J.S.: RPCC-UACJS 2016; 7 (2): 20-32. Disponible en: https://dialnet.unirioja.es/servlet/articulo? codigo $=5925175$ [Consultado el 20 de marzo de 2020].

40. Idoyaga N, Valencia J, Gil de Montes L, Ortiz G. Efectos del Framing y representaciones sociales de epidemias sanitarias: El Caso de la Gripe A. Escritos 
de Psicología 2012; 5 (3): 31-42. Disponible en: http:// scielo.isciii.es/scielo.php?script $=$ sci_abstract\&pi$\mathrm{d}=$ S1989-38092012000300005 [Consultado el 20 de marzo de 2020].

41. Kinsman J. "A time of fear": Local, national, and international responses to a large Ebola outbreak in Uganda. Global Health 2012; 8 (15): 1-12. Disponible en: https:/globalizationandhealth.biomedcentral.com/ articles/10.1186/1744-8603-8-15 [Consultado el 20 de marzo de 2020].

42. Mohammed A, Sheikh TL, Gidado S, Poggensee G, Nguku P, Olayinka A, et al. An evaluation of psychological distress and social support of survivors and contacts of Ebola virus disease infection and their relatives in Lagos, Nigeria: A cross sectional study-2014. BMC Public Health 2015; 15 (824): 1-8. Disponible en: https://www. ncbi.nlm.nih.gov/pubmed/26307047 [Consultado el 17 de abril de 2020].

43. González-Burboa A, Acevedo C, Vera-Calzaretta A, Villaseca-Silva P, Müller-Ortiz H, Páez D, et al. ¿Son efectivas las intervenciones psicológicas para mejorar el control de la Diabetes Mellitus tipo 2 en adultos?: una revisión sistemática y metaanálisis. Rev Med Chile 2019; 147 (11): 1423-36. Disponible en: http://dx.doi. org/10.4067/S0034-98872019001101423 [Consultado el 23 de marzo de 2020].

44. Urzúa A, Vera-Villarroel P, Caqueo-Urízar A, Polanco-Carrasco R. La Psicología en la prevención y manejo del COVID-19. Aportes desde la evidencia inicial. Terapia Psicológica 2020; 38 (1): 103-18. Disponible en: http://teps.cl/index.php/teps/article/view/273 [Consultado el 30 de abril de 2020].

45. Bitanihirwe BK. Monitoring and managing mental health in the wake of Ebola. Commentary. Ann Ist Super Sanita 2016; 52 (3): 320-2. Disponible en: https://www. ncbi.nlm.nih.gov/pubmed/27698289 [Consultado el 15 de abril de 2020].

46. Krause M. (2020, junio). Entrevista a Mariane Krause. Recuperada de https:/www.latercera.com/tendencias/ noticia/mariane-krause-el-control-de-la-pandemia-se-podria-haber-facilitado-si-se-incluia-la-dimension-sicologica/MTOLQIPFMFF5HPIENMY6T2ACRQ/ 